\title{
sciendo
}

\section{Perspectives of digital identity - the case of online education during the COVID-19 pandemic}

\author{
Claudiu VINȚE \\ Bucharest University of Economic Studies, Bucharest, Romania \\ claudiu.vinte@ie.ase.ro \\ Adriana REVEIU \\ Bucharest University of Economic Studies, Bucharest, Romania \\ reveiua@ase.ro \\ Marian DÂRDALĂ \\ Bucharest University of Economic Studies, Bucharest, Romania \\ marian.dardala@ie.ase.ro \\ Viorel LUPU \\ Research \& Development, Online Services SRL, Buzău, Romania \\ viorel.lupu@onlineservices.ro
}

\begin{abstract}
Each individual is unique, and the digital identity associated with a person should model and guarantee this uniqueness and the ability to reliably recognize him/her. In this paper, we review the challenges posed by the sudden transfer of a significant part of the social activity in the online realm, due to the COVID-19 pandemic, with unforeseen consequences on the educational process in particular. If there was a fairly large spectrum of economic activities that were taking place or were facilitated via a digital medium prior to the COVID-19 pandemic, the education system worldwide proved to not have been prepared to provide an effective and consistent response to the going online approach, which was merely a choice, but rather the only embraceable option. In the context of online education, this paper can design a comprehensive and integrated framework for assisting the process of students' digital identity validation, particularly when attending an examination or any other form of academic evaluation conducted remotely. We also consider the integration of digital identity validation with platforms that offer educational content, knowledge evaluation tests, and other elements to which access must be ensured only for authorized users, based on a strict identity identification process to eliminate those who want to defraud or disrupt the functionality of the system.
\end{abstract}

Keywords: digital identity, online education, identity verification, confident assessment.

\section{Introduction}

We live in a social landscape where the need and ability to prove that we are who we assert we are has become the quintessential aspect of the social contract and may underpin many online activities. An increasing number of individual-to-individual, individual-to-authority, or individualto-entity interactions may be established only based on a trustful identity determination process.

Digital identity especially is a concept that has evolved continuously over the past two decades. As Derek O'Halloran pointed out at the World Economic Forum's Annual Meeting (WEF, 2018, p. 5) that we are still learning and adapting to the meaning of "identity in a digital world" syntagm. The current economic and health situation brings to the fore needs that only a few years ago were of little importance. Imposing social distance, working remotely, interacting with 
state or private institutions without physical presence, needing communications, multimedia systems, security, identity, authentication, authorization, procedures, norms, regulations, laws, etc. The new normality requires society as a whole, they adapt quickly, requiring continuous change.

The ubiquity of the Internet opens up new vulnerabilities and opportunities to expose unintentionally protected data, to be unknowingly involved in digital identity theft, fraud, to make erroneous transactions in terms of not adapting workflows to new ways of working (Tobin and Reed, 2017; ITU, 2018). A good example might be a mistake made at home by a bank operator worth about $\$ 900$ million, due to a lack of procedure and attention paid to child custody, but especially due to an inadequate software infrastructure used since 1990-2000 (Ennis, 2020).

Digital identity is the unique representation of a subject engaged in an online transaction. Moreover, the public space is concerned about imposture in the medical field. It is legitimate for the patient to know that the doctor he or she is going to see is not an impostor. The presence of the doctor's identity card does not solve this situation. Many such circumstances can be imagined in which the imposture could be eliminated: in the interaction with public agents of any type. Digital identity is always unique in the context of a digital service, but it does not necessarily need to uniquely identify the subject in all contexts (Grassi et al., 2021). In other words, accessing a digital service may not mean that the real identity of the subject is known. Identity verification establishes that a subject is who he or she claims to be. Digital authentication is the process of determining the validity of one or more authenticators used to claim a digital identity.

Authentication establishes that a subject engaged in trying to access a digital service controls the technologies used for authentication (World Bank Group - GSMA, 2016). Successful authentication provides reasonable assurance based on the risk that the person accessing today's service is the same as the person who previously accessed the service. Digital identity presents a technical challenge because this process often involves checking individuals over an open network and to access digital services, individual subjects must authenticate through open, public networks. There are multiple opportunities (especially in open networks) for identifying theft, and other attacks that fraudulently claim the digital identity of another subject.

As the public increasingly uses electronic services to look for a job, to enrol in an education program, volunteering, etc., a lot of personal data about education, family, and insurance are multiplied and transmitted via the Internet. Many copies of this data are not properly managed by those who request them, and the affected person, although entitled, for example, to request those documents' destruction under GDPR laws, may have completely forgotten about those copies.

It is necessary to set up centralized data warehouses, under the users' control, warehouses managed by professional services, to ensure the rights of users in the Internet environment, but also to certify the correctness of this data. The management services will transmit to the user-designated organizations only that data which is strictly necessary.

\section{Literature review}

Digital identity has become a topic of interest for researchers as the number of publications delivered in the last decade and indexed in well-quoted scientific databases, such as Thomson Reuters and Scopus exceeds 500 papers and 900, respectively. Computer science is the most prominent area and includes half of the published papers.

However, the integration of digital applications in everyday life requires adapted solutions to accurately uncover users' real identities and their relevant specific characteristics (Rivera et al, 2017). Scholars from different areas have emphasised the need for users' authorisation in digital learning and development systems (JISC, 2019), as a precondition for digital citizenship (Chen et 
al., 2021), in health systems because the health data contain sensitive, private information and medical conditions of the patient (Thapa and Camtepe, 2021), in social media activities (Ristoski and Paulheim, 2016). Furthermore, individual protection strategies like management of digital identities, privacy parameters for users' accounts, and blockers' settings have stimulated economic activities delivered online (Kaaniche, 2020).

The emergence of The Internet of Agreements (World Government Summit, 2017), which connects digital content delivered through the Internet and real-world contracts and regulations, enables the next generation of digital business, where user authentication over the network plays a key role and has fostered new types of legal documents. Furthermore, the IoT technology (Internet of Things) enables computers to organize and manage things that need to be uniquely identified through digital identity (Tavana et al., 2020; Ahmed et al., 2019).

Various solutions have been already proposed to identify digital users and digital things connected to the Internet. User authentication by identifying the individual's physiological or behavioural attributes based on biometric technology has provided good results as reported by (Karnana et al., 2011), among others. To a large extent, the available solutions involve smart cards as a powerful support for digital identification, due to their ability to provision information management through multiple applications, as well as transaction processing on a single card (Shelfer et al., 2004).

More recently, the blockchain, a protocol ensuring that the information remains intact over time, has been introduced as an underpinning solution to manage users' digital identity, to distinguish between different entities, and to support distributed user authentication (Liu et al., 2020).

\section{Proposed framework for identity validation in the context of online examination}

This section introduces a new architectural solution that integrates students' identity validation in three scenarios used for summative assessment activities occurring in the educational context generated by the pandemic, when both educational activities and students' evaluation have to be conducted online. The framework proposed in this research paper is in the development phase and we have conducted tests within the development environment, up to this point in time. The emerging solution is going to be deployed in a near production environment and tested on a sample of students from the Bucharest University of Economic Studies, Romania.

\section{Challenges and working scenarios for conducting the online assessment}

The circumstances considered for our research envisage assessment activities conducted online, with students connected to a remote location and able to use the following minimum resources: an Internet connection, a smartphone, a computer, a video camera and a microphone.

Based on our teaching experience, we have identified the following challenges and premises of the interaction between the student and the examiner that have to be considered and handled during an online examination activity:

1. The student who takes the individual assessment can be in the same physical location with a group of other students, or third parties who can provide undue support.

2. The student is not necessarily interested in the process of identity verification and access authorization to be correct. In other words, there is no consensus, a common interest of both parties, the university on the one hand and the student on the other hand, that this process allows the student to be evaluated and only the expected student accesses the platform and resources during the evaluation process. 
3. The student will participate in the evaluation process through a video conferencing session, conducted by Zoom, Cisco Webex, Meet Google etc.

4. The student will be evaluated through the blended learning online.ase.ro platform or using a local IDE application - required for assessing the programming subjects, when the code created by the student will be future uploaded on online.ase.ro platform.

5. Each student is assumed to have access to a smartphone running Android or IOS, with or without NFC (Near Field Communication) support capabilities.

6. If the student may use two video cameras placed against each other, she or he will be located in between the two cameras (optional).

Multiple examination options for online assessments are available and have been taken into consideration for our proposed framework. For our research purpose, it is important to have a detailed description of the interaction between the human (the student) and the system (considering the different hardware components involved) because the possible shortcomings can be easily revealed.

Online evaluation scenario 1 - Individual evaluation - through a set of open-ended questions and under time constraints.

Currently, the student-educational platform interaction supposes to follow the steps:

1.1. The student connects to the platform using his/her username and password.

1.2. The availability of the link associated with the exam proves that the student receives the right to attend the exam.

1.3. Based on the individual link, the student will receive a topic that is randomly extracted from a pool of questions.

1.4. The student may download the subject statement from the platform;

1.5. The student has a predetermined time span to solve the subject; depending on the discipline, she/he has to enter the code via a local IDE, installed on the student's computer (in the case of programming discipline), or the student has to write the solution on the paper (in the case of subjects like mathematics, statistics, etc.). In both situations, the student's outcome will be uploaded on the platform within the time allocated for the exam, using the student's own credentials.

1.6. Throughout the examination process, the student is connected to the examining teacher via an audio-video conferencing session.

Online evaluation scenario 2. Individual examination usually involves rather complex problems and under time constraints. This scenario includes two parts: firstly, the student independently designs her/his solution and uploads it to an educational platform, and then follows an online individual conversation between the student and the evaluator.

2.1. - 2.6 The corresponding steps are followed similarly to scenario 1;

2.7. In addition, a one-to-one oral examination is conducted via a video conferencing platform; the teacher has a face-to-face discussion with the student about the solution that the latter uploaded on the platform. At least two aspects should be noted:

2.7.1. Students who enter this oral exam later have more time to "rethink" their solution; even if what they uploaded as a solution can be found on the platform, they can "argue" that they knew the correct answer but made mistakes; of course, the examiner can ask questions from the topics that were not necessarily in the subject, but it appears "unfair" 
to deviate too much from the subject that the student received at the beginning of the examination.

2.7.2. In conjunction with the previous aspect, the assessment process is extremely stressful and time consuming, rather than for the examining teacher.

Online evaluation scenario 3. Individual examination using a quiz-based evaluation that includes some multiple-choice questions, randomly selected from a large set of questions, managed so that the student cannot review, modify, or return to the previous question and only one question is provided on the screen at the given time. The evaluation is conducted via an educational platform. 3.1. The student accesses the eLearning platform using her/his own credentials (username and password).

3.2. Each student accepted for the examination will access the individual assessment quiz with a lot of multiple-choice questions. As these questions are drawn at random from a larger crowd of questions and the answers to the questions are amalgamated, each student will have a unique quiz to solve. The student's interaction during the examination is only with the educational platform; she/he does not need to use a local IDE or to provide a hand-written answer.

3.3. Computer programming related quizzes usually contain portions of the code and the students are asked to identify what would be obtained after running that particular piece of code; in the case of mathematics and statistics subjects, for example, the quiz can contain problems with calculable / detectable results.

3.4. Throughout the evaluation, the student is connected via a video conferencing session with the examining teacher.

3.5. At the end of the quiz, the student receives the evaluation outcome automatically generated by the eLearning platform, as well as the feedback on the correct/wrong answers.

From the perspective of conducting an online evaluation, if this scenario is accompanied by the student's identity validation and if the student has only one connection available to access the educational platform during the evaluation period, it can be considered as a reliable context for reducing the risk of exam fraud.

The first two scenarios have the advantage of consenting a rather comprehensive evaluation form, and they are appropriate for different subjects with various evaluation purposes. However, the assessment activities require a longer time span than in the case of the third scenario, and they are not suitable when a big number of students need to be evaluated.

A common challenge in all online scenarios is the validation of the students' identity before the examination. Currently, this is done by checking an official document issued by the Romanian authorities, such as an identity card, a driving license, a passport or, within an organizational environment, ID cards of any kind. These documents are made for autonomous use, possibly with limited validation, such as passports, and without a holistic, unitary vision in line with the ambitions of the information or knowledge-based society. Such documents contain personal data that cannot be validated in real time by the authority that issued the document. The use of stand-alone documents, such as identity cards in the Internet environment, involves risks for the person concerned. It is often not necessary to transmit the data contained in the identity card, but they are often collected abusively by the electronic service operators. Identity documents cannot certify other qualities held by the holder. 


\section{Proposed architectural framework}

With all three scenarios in mind, we propose an architectural framework to improve the student's identity validation in a summative assessment setting, with the subsequent goal to diminish academic malpractices.

The digital identification component is involved in all three aforementioned scenarios to validate the students' identity before and during the evaluation session, like a component of the student-teacher- eLearning system interaction, as presented in Figure 1.

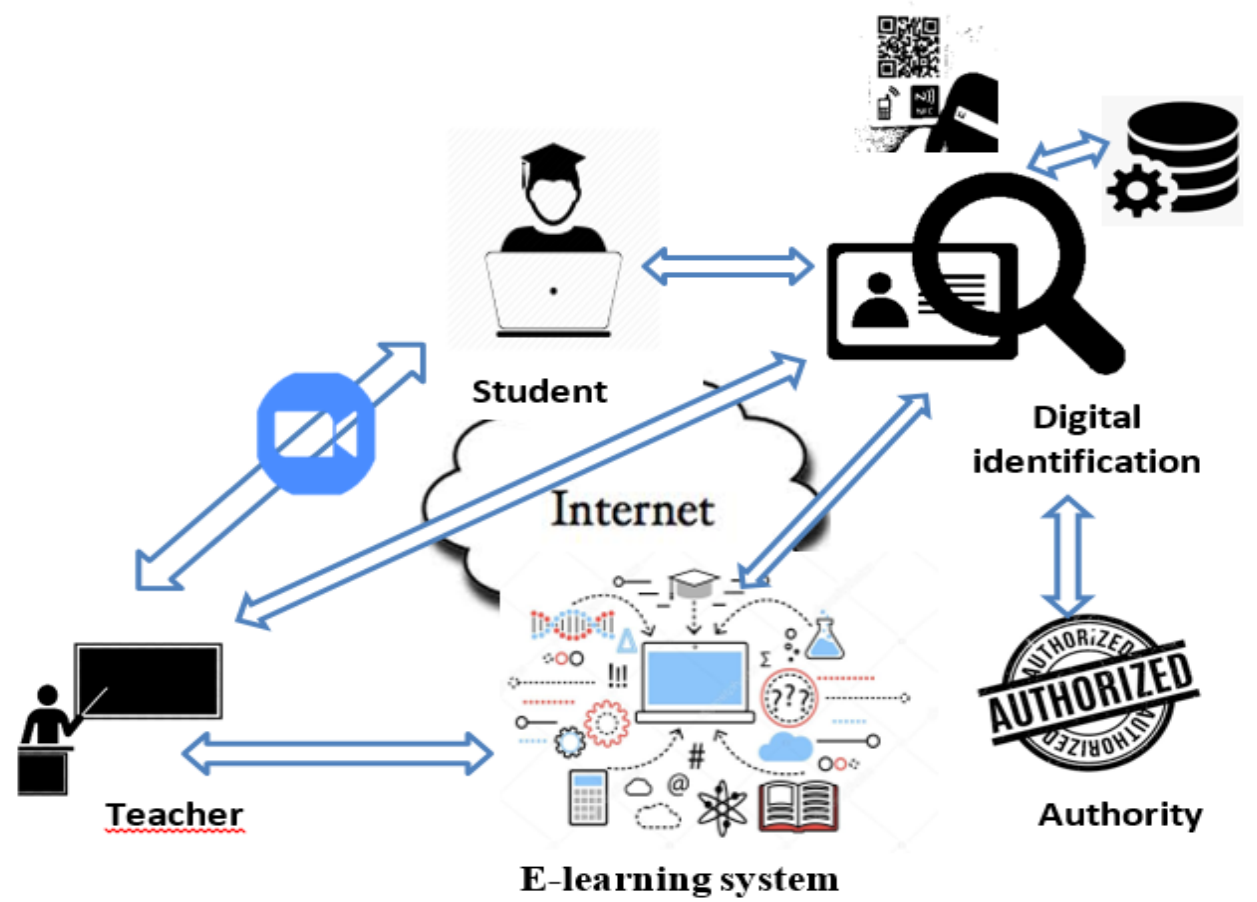

Figure 1-The architecture of the online evaluation system including digital authentification

There is a significant number of technological innovations useful to support digital authentication (Okada et al., 2019), those appropriate in the online eLearning context being considered in our research and depicted in Figure 2.

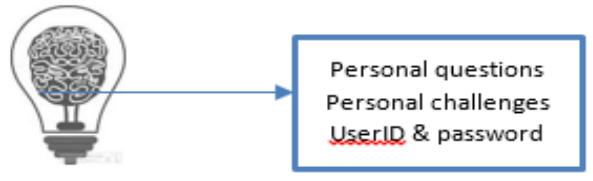

Knowledge

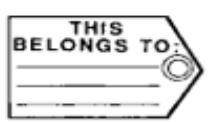

Possession

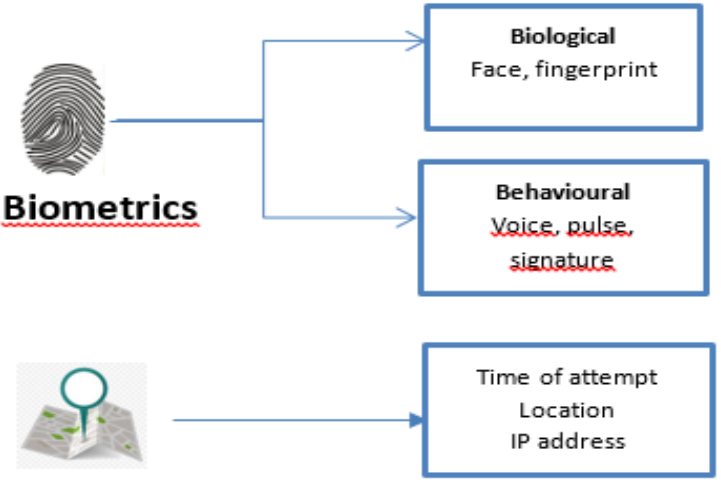

Mechanisms

Figure 2- Instruments for digital identification in online eLearning 
Knowledge-based authentication tools provide a low level of security, but are easy-to-use, inexpensive and are commonly used in most of the online eLearning systems.

Biometrics solutions use physiological and behavioural individual characteristics to effectively and accurately recognise the student, although they can be technically complex and expensive (Okada et al., 2019).

Possession instruments include hardware devices with or without some specific facilities, as is the NFC technology elected for our system, that are definitely in the user's possession or that can be delivered at a reasonable cost.

Mechanisms provide additional information generated by the user's connection to the local network or the Internet that can be meaningfully combined with other personal information during digital authentication.

NFC technology was elected and implemented to support the digital identification of students so far, because it is available with most of the actual smartphones and it is almost an inexpensive solution, so suitable for an online eLearning system, as to a large extent students own at least one smartphone device.

Moreover, the NFC devices allow to assign a unique identifier to each user and to manage the rest of the students' details via a database, to gather data from the database and to display only the necessary data, identified based on the specific educational context (e.g. subject, type of assessment). In this way, the only needed filtered data is presented to the requesting user in a confidential manner. A Quick Response (QR) code is generated based on the NFC address that may be printed on the front of a physical or virtual card and can be used in certain conditions, when a device with access to the NFC technology is not available, e.g. smartphones or tablets that do not include an NFC reader. Furthermore, the use of custom NFC readers allows the development of specific applications, which provide additional facilities for the authenticated users and are compatible with mobile devices (smartphones or NFC-enabled tablets) for the public environment.

Within the development environment, we have successfully tested the following four-step process for digital identity verification. As a perquisite, the student has to have a valid phone number registered with the university, as part of the academic education contract.

1. The student receives on his or her institutional email account a digital ID card, containing, among other personalized information, a QR code;

2. Within the video conference session designated for an online examination, the student is asked by the teacher to present a digital ID card, potentially from his or her smartphone or a shared computer screen;

3. The teacher scans the QR code within the web browser and receives the student's identification data from the server side of our proposed solution;

4. Based on the teacher's approval, the system makes a phone call to the student's phone number and supplies via a voice-based assistant the necessary credentials for allowing the student to authenticate against the university's blended learning platform, to enter the online examination.

The digital identification component is embedded in all three online evaluation scenarios, whenever the student has to interact with the eLearning system, except for the direct video-audio connection between the student and the teacher, as depicted in Figure 1.

For institutional access, the proposed identification element used so far, is a QR code sent to the student for anonymized identification. This code is generated by the service provider to connect the student ID with relevant information such as: a picture for visual identification in a video conferencing system, personal information for identifying the person by direct interrogation in relation to some information that is likely to be said instantly, and student track during class. 


\section{Conclusive remarks and further research}

The long-term vision of this ongoing research is to integrate other biometrics and location-based mechanisms to support users' identification as well as to create a distributed, fault-tolerant and permanently available system, assisted by an economic structure and services that allow but are not limited to: protection of personal data in accordance with current legislation, European Union recommendations and international best practices; profiling access to personal data according to the characteristics of the requesting entity, such as "need to know basis"; to control of personal data will be driven by the data owner; to impersonate, digital identity generation, association with physical identities, digital identity profiling; services for identity consultation, authentication services, services for federalization of authentication.

Using open standards for systems' integration, the digital identification component will be connected with various platforms that offer learning content, knowledge evaluation tests, and other elements to which access must be ensured only for authorized persons based on a strict identity identification process, to eliminate those who want to defraud or disrupt the functionality of the system. A significant advantage of the proposed framework is the flexibility to be adapted based on the field of interest to which digital identification is required; the system will extract the relevant information for the digital identification so that the functionality is not harmed by false actors.

\section{Acknowledgment}

This work was supported by the project co-financed by the European program POC, 2014-2020, entitled Identity management solution and advanced authentication using converged technologies and ensuring higher levels of security for access to critical applications and platforms: LegitimID (MySMIS project code: 129617).

\section{References}

Ahmed, A.I.A., Ab Hamid, S.H., Gani, A., Khan, S., Khan, M.K. (2019). Trust and reputation for Internet of Things: Fundamentals, taxonomy, and open research challenges, Journal of Network and Computer Applications, 145.

Chen, L.L., Mirpuri, S., Rao, N., Law, N. (2021). Conceptualization and measurement of digital citizenship across disciplines, Educational Research Review, vol. 33.

Ennis, D. (August 2020). Citi cites human error in accidental \$900M transfer. Banking Dive: https://www.bankingdive.com/news/citi-cites-human-error-in-accidental-900m-transfer/ 584156/.

Grassi, P.A., Garcia, M.E., \& Fenton, J.L. (2021). Digital Identity Guidelines, National Institute of Standards and Technology, NIST Special Publication, 800-63-3.

International Telecommunication Union, ITU (2018). Digital Identity Roadmap Guide. Creative Commons Attribution 3.0 IGO (CC BY 3.0 IGO), ITU Geneva, Switzerland.

Joint Information Systems Committee (JISC, 2019). Digital capabilities framework: the six elements defined, http://repository.jisc.ac.uk/7278/1/BDCP-DC-Framework-Individual6E-110319.pdf.

Karnana, M., Akila, M., Krishnaraj, N. (2011). Biometric personal authentication using keystroke dynamics: A review, Applied Soft Computing, 11, 1565-1573.

Kaaniche, N., Laurent, M., \& Belguith, S. (2020). Privacy enhancing technologies for solving the privacy-personalization paradox: Taxonomy and survey, Journal of Network and Computer Applications, vol. 171. 
Liu, Y., He, D., Obaidat, M.S., Kumar, N., Khan, M.K., Choo, K.K.R. (2020). Blockchain-based identity management systems: A review, Journal of Network and Computer Applications, vol. 166.

Okada, A., Whitelock, D., Holmes, W., \& Edwards, C. (2019). e-Authentication for online assessment: A mixed-method study, British Journal of Educational Technology, 50(2), 861-875.

Ristoski, P., \& Paulheim, H. (2016). Semantic Web in data mining and knowledge discovery: A comprehensive survey, Journal of Web Semantics, Vol. 36, 1-22.

Rivera, R., Robledo, J.G., Larios, V.M., Avalos, J.M. (2017). How digital identity on blockchain can contribute in a smart city environment. In: 2017 International Smart Cities Conference (ISC2), 1-4.

Shelfer, K.M., Corum, C., Procaccino, J.D., \& Didier, J. (2004). Smart Cards, Advances in Computers, 147-192.

Tavana, M., Hajipour, V., \& Oveisi, S. (2020). IoT-based enterprise resource planning: Challenges, open issues, applications, architecture, and future research directions, Internet of Things.

Thapa, C., \& Camtepe, S. (2021). Precision health data: Requirements, challenges and existing techniques for data security and privacy, Computers in Biology and Medicine, vol. 129.

Tobin, A., \& Reed, D. (March 2017). The Inevitable Rise of Self-Sovereign Identity. A white paper from the Sovrin Foundation.

WEF (2018). World Economic Forum, Davos Identity in a Digital World. A new chapter in the social contract. Cologny/Geneva, Switzerland.

World Bank Group - GSMA - Secure Identity Alliance Discussion Paper (2016). Digital Identity: Towards Shared Principles for Public and Private Sector Cooperation, July 2016, Washington DC.

World Government Summit (2017). Building the hyperconnected future on blockchains, http://internetofagreements.com/files/WorldGovernmentSummit-Dubai2017.pdf. 